\title{
Obesidad, depresión y factores asociados a la calidad de vida en artroplastia total de rodilla
}

\section{Obesity, depression and factors associated to the quality of life in total knee arthroplasty}

\author{
Sergio Charles-Lozoya ${ }^{1 *}$, Héctor Cobos-Aguilar², Juan C. Tamez-Montes ${ }^{1}$, Jesús M. Brizuela-Ventura ${ }^{1}$, \\ Jesús M. Rangel-Valenzuela ${ }^{3}$ y Adrián García-Hernández ${ }^{1}$ \\ ${ }^{1}$ Servicio de Artroplastia de Rodilla, División de Educación en Salud, Hospital de Traumatología y Ortopedia No. 21, Instituto Mexicano del Seguro \\ Social, Universidad de Monterrey, Monterrey, Nuevo León, México; '2División de Ciencias de la Salud, Universidad de Monterrey, Monterrey, Nuevo \\ León, México; ${ }^{3}$ Baylor University, Texas, Estados Unidos
}

\section{Resumen}

Antecedentes: La gonartrosis puede acompañarse de depresión y obesidad, afectando la calidad de vida (CV) y la funcionalidad después de una artroplastia total de rodilla (ATR). Objetivo: Comparar la CV en sujetos con depresión y obesidad 5 años después de una ATR. Método: Estudio transversal prolectivo, con dos y tres grupos basados en el estatus de depresión y la obesidad. Se obtuvieron variables sociodemográficas, clinimétricas, antropométricas y de CV. La depresión se evaluó con el cuestionario de tamizaje del Estudio Nacional sobre Salud y Envejecimiento de México (CENASEM). La CV se evaluó con la encuesta de salud SF-36. Resultados: Se revisaron 378 participantes, para incluir 266. Se detectó depresión en el 24.1\%, mujeres $(p<0.001)$, con comorbilidad $(p=0.04)$ y ansiedad $(p<0.001)$. El puntaje en la CV en sujetos con depresión fue menor $(p<0.001$ ). Las personas obesas tuvieron mayor dolor (escala visual análoga 3 vs. $2 ; p=0.002$ ) y afección del dominio de vitalidad en la CV (75 vs. 80; $p=0.02$ ). Conclusiones: Es indispensable evaluar la depresión y la CV con cuestionarios como el CENASEM y el SF-36 en ancianos que se sometan a ATR, ya que detectar y tratar la depresión podría aumentar la CV y la función. Las personas ancianas obesas con ATR pueden presentar mayor dolor.

Palabras Clave: Artroplastia. Calidad de vida. Depresión. Índice de masa corporal. Obesidad.

\begin{abstract}
Background: Knee osteoarthritis can be accompanied by depression and obesity, which could affect the quality of life (QOL) and function after a total knee arthroplasty (TKA). Objective: To compare the QOL in subjects with depression and obesity 5 years after TKA. Methods: Cross-sectional study of prospectively collected data, with two and three groups based on depression and obesity status. Sociodemographic variables, QOL and pain were compared. Depression was evaluated with a screening geriatric depression questionnaire used in the Mexican Health and Age Study (CENASEM). QOL was evaluated with the health survey SF-36. Results: 378 participants were reviewed, 266 were included. Depression was detected in $24.1 \%$, female $(p<0.001)$, with comorbidity $(p=0.04)$ and anxiety $(p<0.001)$, and the $Q O L$ score in subjects with depression was lower $(p<0.001)$. Obese subjects had greater pain (analogous visual scale 3 vs. 2; $p=0.002$ ) and affection of the vitality domain
\end{abstract}

\footnotetext{
Correspondencia:

*Sergio Charles-Lozoya

Avda. José Ma. Pino Suárez y Juan Ignacio Ramón, s/n

Col. Centro

C.P. 64000 , Monterrey, N.L., México

Fecha de recepción: 23-12-2018

E-mail: scharleslozoya@yahoo.com.mx;

sergio.charlesl@imss.gob.mx

DOI: 10.24875/CIRU.19000989

Cir Cir. 2020;88(2):143-149

Contents available at PubMed www.cirugiaycirujanos.com 0009-7411/@ 2019 Academia Mexicana de Cirugía. Publicado por Permanyer. Este es un artículo open access bajo la licencia CC BY-NC-ND (http://creativecommons.org/licenses/by-nc-nd/4.0/).
} 
in the QOL (75 vs. 80; $p=0.02$ ). Conclusions: Evaluating depression and QOL with questionnaires such as CENASEM and SF-36 in elderly subjects who undergo ATR should be indispensable, since detecting and treating depression could increase QOL and function. Obese elderly with TKA may present greater pain.

Key Words: Arthroplasty. Quality of life. Depression. Body mass index. Obesity.

\section{Introducción}

La artroplastia total de rodilla (ATR) es un tratamiento eficaz para la artritis de la rodilla en etapa avanzada, ya que proporciona alivio del dolor y mejora la calidad de vida $(C V)^{1,2}$. Se estima que la prevalencia de ATR en adultos de 50 años o más es del $4.2 \%^{3}$, lo que corresponde a 4.7 millones de reemplazos $^{4}$. Aunque la ATR alivia el dolor, el $7-34 \%$ de los pacientes no están satisfechos con el resultado global de la cirugía 5 . Así mismo, los sujetos que se someten a ATR sufren comorbilidad que puede impactar en la recuperación y limitar los resultados funcionales, así como la $\mathrm{CV}^{6}$. Los factores implicados en esta insatisfacción son el sexo, el estatus económico, la comorbilidad, la salud psicológica, el estatus marital y el apoyo social, que si están alterados antes de la cirugía podrían ser predictores de resultados inadecuados de la ATR, tanto en la CV como en la función? Uno de estos factores es la depresión ${ }^{8}$, que puede asociarse con dolor persistente e ingesta constante de medicamentos para tratarlo9. Así mismo, la depresión influye en los resultados funcionales de la ATR de forma independiente de la presencia o la intensidad del dolor ${ }^{10}$. Del mismo modo, el estrés mental, al generar emociones negativas, se asocia con pobres resultados funcionales, afección de la CV, discapacidad y dolor agudo recurrente ${ }^{11}$.

Por otro lado, la obesidad es una epidemia global con implicaciones sociales, médicas y financieras. Se estima que hay más de 502 millones de personas clasificadas con obesidad en el mundo ${ }^{12}$, lo que aumentará la demanda de ATR en un 673\% para el año 2030. Ante este incremento, se hace obligatoria su evaluación adecuada para evitar resultados insatifactorios ${ }^{13}$. Se refiere que después de 2 años de una ATR en sujetos obesos el peso aumenta, ya que la obesidad es un problema multifactorial y el simple alivio del dolor no es suficiente para mejorar la actividad física que disminuya el índice de masa corporal (IMC) y aumente la CV ${ }^{14}$.

De esta manera, los estudios que evalúan la obesidad y la depresión, así como su influencia en los resultados de la ATR, son discordantes y aún no existe un consenso que evalúe la CV, la función y la satisfacción en forma homogénea, por lo que se generan resultados contradictorios y discrepantes. Por lo tanto, el objetivo principal de este estudio fue comparar la CV en pacientes con obesidad y depresión 5 años después de una ATR.

\section{Método}

Encuesta transversal prolectiva, realizada en un hospital regional especializado en traumatología y ortopedia del Instituto Mexicano del Seguro Social del Noreste de México. Los criterios de inclusión fueron pacientes operados de ATR entre 2010 y 2012, con más de 5 años poscirugía, mayores de 50 años, sin tratamiento antidepresivo ni ansiolítico, y que no presentaran deterioro cognitivo. Los criterios de exclusión fueron sujetos con reemplazo en otras articulaciones, cirugías de revisión o por tumoración, artritis inflamatorias, fibrosis articular, infección previa, artrodesis y aflojamiento aséptico. La selección muestral se hizo a partir del registro quirúrgico diario de ATR, con muestreo probabilístico aleatorio simple sin reemplazo y tamaño muestral para proporción, en población finita (2,500 ATR al año), con una frecuencia esperada de depresión del $11.7 \%{ }^{15} \mathrm{y} \mathrm{d}=0.01$, para obtener 266 pacientes.

A los participantes se les contactó telefónicamente para su evaluación, y previo consentimiento informado se expuso el objetivo del estudio para registrar la edad, el sexo, la escolaridad ( $\leq 6$ años $0>6$ años), el estado civil (casado o no casado), el tabaquismo y la ingesta de alcohol, así como la presencia de diabetes mellitus, hipertensión arterial sistémica, comorbilidad (padecimientos pulmonares, cardiovasculares, urológicos, renales, hepatopatías, cáncer, endocrinopatías y enfermedad cerebral), ingesta de antiinflamatorios no esteroideos, jubilación reciente, ocupación y la principal fuente económica (pensión, familia o por trabajo). El peso y la talla se tomaron con ropa ligera, sin zapatos, en una báscula analógica con estadímetro y verificación de calibración; se registraron en dos ocasiones y se tomó la media como la medición definitiva, para después calcular el IMC $\left(\mathrm{kg} / \mathrm{m}^{2}\right)$ y categorizar en tres grupos: $\leq 24.9$ (peso normal), $25-29.9$ (sobrepeso) y $\geq 30$ (obesidad). 
Los instrumentos se aplicaron en dos ocasiones por personal ajeno a la investigación (estudiantes de medicina previamente entrenados), en forma ciega e independiente, al entrar y salir de la consulta; además, se estimó la concordancia entre observadores. Para detectar la depresión se usó el cuestionario de tamizaje del Estudio Nacional sobre Salud y Envejecimiento de México (CENASEM) ${ }^{16}$, sensible $(80.7 \%$ ) y específico (68.7\%), consistente (alfa de Cronbach: $0.74)$, conformado por nueve reactivos con respuestas sí/no; con seis respuestas positivas y tres negativas, valora los eventos en la última semana en cada pregunta. Un puntaje de 5 o más indica depresión. La CV se evaluó con el cuestionario SF-36 (CSF-36), que consta de 36 preguntas y 8 dimensiones: función física, rol físico, dolor corporal, salud general, vitalidad, función social, rol emocional y salud mental. Es autoadministrado, los resultados se trasladan a una escala de 0 (peor salud) a 100 (mejor salud), y tiene un alfa de Cronbach de 0.56 a $0.84^{17}$. La intensidad del dolor se midió con una escala visual analógica (EVA) de 0 a 10, en la que 0 era ausencia y 10 era la máxima intensidad de dolor. El paciente señaló el número que mejor indicase esa intensidad. Es una escala confiable $(r=0.94)$, útil incluso en analfabetos $(r=0.71)^{18}$. Se aplicó dos veces y se tomó la mediana como la definitiva. La ansiedad se evaluó con el cuestionario de Goldberg con dos subescalas (depresión y ansiedad), cada una con nueve ítems de respuesta sí/no, con cuatro iniciales de tamizaje y los otros cinco que se formulan al obtener respuestas positivas al tamizaje. Es sensible (83.1\%) y específico (81.8\%), con un alfa de Cronbach de $0.65^{19}$. Para el estudio solo se aplicó el cuestionario de ansiedad.

Para la correcta aplicación y llenado de los instrumentos de evaluación se realizaron varias reuniones con el psiquiatra del hospital, así como supervisión semanal a los colaboradores y una prueba piloto antes de iniciar el estudio. El protocolo fue autorizado por el Comité Local de Investigación y Ética en Investigación 1903, con registro R-2017-1903-10.

Se utilizaron las pruebas estadísticas $U$ de Mann-Whitney y Kruskal-Wallis para variables cuantitativas, y la prueba de ji al cuadrado de Pearson para la comparación de frecuencias. Para la magnitud de la asociación se calculó la razón de momios, con un intervalo de confianza del 95\%. Se realizó un análisis multivariado mediante un modelo de regresión logística para discriminar aquellos factores que en el análisis bivariado fueron significativos. Se consideró significativo un valor de $p<0.05$. El análisis se realizó con el programa SPSS versión 22 (SPSS, Chicago, IL, USA).

\section{Resultados}

Se evaluaron 378 sujetos, de los que 112 no cumplieron los criterios de selección (23 con reemplazo articular de cadera, 16 con prótesis de revisión, 13 con antecedente de aflojamiento protésico, 9 con artrodesis, 7 con prótesis tumoral, 12 que reportaron ansiedad y 32 con depresión), para ingresar finalmente 266 participantes. La mediana de edad fue de 71 años (rango: 51-89). Preponderantemente eran mujeres $(61.7 \%)$, y en el $51.9 \%$ el IMC fue $\geq 30 \mathrm{~kg} / \mathrm{m}^{2}$. La prevalencia de depresión y de ansiedad fue del $24.1 \%$ y del $17.7 \%$, respectivamente. En la comparación de las variables en sujetos con ATR clasificados con y sin depresión, el sexo femenino, la ansiedad, la ingesta de alcohol y la comorbilidad fueron significativas (Tabla 1). Los sujetos clasificados con depresión obtuvieron puntuaciones bajas en todos los dominios del CSF-36, con diferencias significativas con respecto a los sujetos sin depresión $(p<0.001)$ (Tabla 2). En el análisis comparativo por sexo, las mujeres tuvieron una prevalencia de depresión y de ansiedad del $84.4 \%$ y del $74.5 \%$, respectivamente. En los resultados de sujetos diabéticos con ATR se obtuvo una mediana de tiempo de evolución de la diabetes de 8 años (rango: 1 - 33), en el $25.9 \%$ el tratamiento era con hipoglucemiantes, el $4.9 \%$ usaban insulina y solo el dominio de salud general del CSF-36 mostró una puntuación baja $(p=0.009)$. En los sujetos con hipertensión arterial sistémica, el dominio de función social fue significativo $(p=0.03)$. En los participantes clasificados con ansiedad, en los ocho dominios del CSF36 hubo puntuaciones bajas $(p<0.001)$, pero en la EVA no hubo diferencias significativas. A su vez, en la comparación realizada entre los grupos de sujetos con peso normal, sobrepeso y obesidad, con el CSF36 , los componentes de función física, dolor corporal, vitalidad y EVA tuvieron significancia estadística (Tabla 3). En el análisis multivariado mediante un modelo de regresión logística binaria, los factores independientes asociados a depresión en los sujetos ancianos con ATR fueron la ansiedad, la función social, la vitalidad y la salud mental (Tabla 4).

\section{Discusión}

En este estudio se pudo observar una prevalencia elevada de depresión y ansiedad en ancianos con 
Tabla 1. Comparación de las características generales de la población de acuerdo con el estatus con y sin depresión

\begin{tabular}{|c|c|c|c|c|c|}
\hline Característica & Con depresión, $\mathrm{n}=64$ (24.1\%) & Sin depresión, n = 202 (75.9\%) & $\mathrm{RM}$ & IC 95\% & $\mathrm{p}$ \\
\hline Edad (años) & $70.5(63.25-76)^{\star}$ & $71(66-75)^{*}$ & - & - & $0.61^{\dagger}$ \\
\hline Sexo femenino (\%) & $54(84.4)$ & $110(54.5)$ & 4.5 & $2.2-9.4$ & $<0.001^{\ddagger}$ \\
\hline Peso (\%) & $75.5(68-87.3)^{\star}$ & $79.5(72-86)^{*}$ & - & - & $0.16^{+}$ \\
\hline Talla (\%) & $1.6(1.53-1.61)^{\star}$ & $1.6(1.55-1.68)^{*}$ & - & - & $0.06^{+}$ \\
\hline Escolaridad $\leq 6$ años (\%) & $7(10.9)$ & $20(9.9)$ & 1.1 & $0.4-2.8$ & $0.81^{\ddagger}$ \\
\hline Estado civil no casado (\%) & $23(35.9)$ & $55(27.2)$ & 0.7 & 0.4-1.2 & $0.18^{\ddagger}$ \\
\hline Tabaquismo (\%) & $5(7.8)$ & $26(12.9)$ & 0.6 & $0.2-1.6$ & $0.27^{\ddagger}$ \\
\hline Ingesta de alcohol (\%) & $6(9.4)$ & $47(23.3)$ & 0.3 & $0.1-0.8$ & $0.02^{\ddagger}$ \\
\hline Diabetes mellitus (\%) & $23(35.9)$ & $62(30.7)$ & 1.3 & $0.7-2.3$ & $0.43^{\ddagger}$ \\
\hline Hipertensión arterial sistémica (\%) & $41(64.1)$ & $124(61.4)$ & 1.1 & $0.6-2.0$ & $0.70^{\ddagger}$ \\
\hline Comorbilidad (\%) & $39(60.9)$ & $93(46)$ & 1.8 & $1.03-3.2$ & $0.04^{\ddagger}$ \\
\hline Ansiedad (\%) & $32(50)$ & $15(7.4)$ & 12.5 & $6.0-25.5$ & $<0.001^{\ddagger}$ \\
\hline Uso de AINE (\%) & $51(79.7)$ & $141(69.8)$ & 1.7 & $0.9-3.3$ & $0.12^{\ddagger}$ \\
\hline Jubilación reciente (\%) & $10(15.6)$ & $38(18.8)$ & 0.8 & $0.4-1.7$ & $0.56^{\ddagger}$ \\
\hline Trabaja (\%) & $10(15.6)$ & $19(9.4)$ & 1.8 & $0.8-4.1$ & $0.16^{\ddagger}$ \\
\hline Soporte económico (\%) & & & & & $0.96^{\ddagger}$ \\
\hline Laboral & $15(23.4)$ & $25(12.4)$ & - & - & \\
\hline Pensión & $43(67.2)$ & $157(77.7)$ & - & - & \\
\hline Familia & $6(9.4)$ & $20(9.9)$ & - & - & \\
\hline
\end{tabular}

AINE: antiinflamatorios no esteroideos; IC 95\%: intervalo de confianza del 95\%; RM: Razón de momios.

${ }^{*}$ Mediana y amplitud intercuartil.

+U de Mann-Whitney.

‡Ji al cuadrado.

Tabla 2. Comparación de los componentes de la escala SF-36 de acuerdo con el estatus con y sin depresión

\begin{tabular}{|c|c|c|c|}
\hline Componente & $\begin{array}{l}\text { Con depresión, } \\
n=64(24.1 \%)\end{array}$ & $\begin{array}{l}\text { Sin depresión, } \\
n=202(75.9 \%)\end{array}$ & $p$ \\
\hline Función física & $62.5(50.5-83.75)$ & $80(70-90)$ & $<0.001^{*}$ \\
\hline Rol físico & $25(25-50)$ & $75(25-80)$ & $<0.001^{*}$ \\
\hline Dolor corporal & $52(32.75-61)$ & $73(51-84)$ & $<0.001^{*}$ \\
\hline Salud física & $47(35.5-60)$ & $65(52-80)$ & $<0.001^{*}$ \\
\hline Vitalidad & $60(50-68.75)$ & 82 (72-90) & $<0.001^{*}$ \\
\hline Función social & $54(50-75)$ & $100(75-100)$ & $<0.001^{*}$ \\
\hline Rol emocional & $66(33-100)$ & $100(92-100)$ & $<0.001^{*}$ \\
\hline Salud mental & $62.5(48-76)$ & 92 (84-96) & $<0.001^{*}$ \\
\hline EVA & $3(2-4)$ & $2(1-4)$ & $<0.001^{*}$ \\
\hline
\end{tabular}

Todos los valores están expresados en mediana y amplitud intercuartil.

EVA: escala visual analógica.

*U de Mann-Whitney.

ATR. En contraste, Stundner, et al..$^{20}$, en sujetos antes de ser operados de ATR, reportan una prevalencia del 1.8\% para depresión, del 3\% para ansiedad y del $0.07 \%$ para ambas, lo que refleja que estas alteraciones psiquiátricas podrían volverse más prevalentes después de 5 años de la ATR. Esto coincide con los resultados de Singh y Lewallen ${ }^{21}$, quienes hallaron que después de 13 años existe un aumento en la prevalencia de depresión y ansiedad del 4.1 al $14.8 \%$ y del 4.1 al $8.9 \%$, respectivamente, lo cual podría explicarse por la presencia de dolor persistente que puede propiciar insatisfacción y alteraciones psicológicas. Del mismo modo lo observaron Dhurve, et al., ${ }^{22}$ quienes referir que el dolor persistente y la presencia de depresión después de 3 años de la ATR es la principal causa de insatisfacción; además, los sujetos que presentan depresión antes de ser operados de ATR se asocian con niveles más altos de dolor a 1 año del posoperatorio ${ }^{23}$. Khatib, et al. ${ }^{24}$ refieren que los sujetos con depresión y que están en lista de espera para ATR reportan niveles de dolor más altos, incluso con el mismo grado de degeneración articular. 
Tabla 3. Comparación de los componentes de la escala SF-36 con el índice de masa corporal

\begin{tabular}{|c|c|c|c|c|}
\hline Componente & Peso normal, $\mathrm{n}=20$ (7.5\%) & Sobrepeso, $n=108(40.6 \%)$ & Obesidad, $n=138$ (51.9\%) & $p$ \\
\hline EVA & $3(1-4)$ & $2(1-4)$ & $3(2-4)$ & $0.002^{*}$ \\
\hline Función física & $72.5(52.5-80)$ & $80(70-90)$ & $75(60-85)$ & $0.01^{*}$ \\
\hline Rol físico & $50(25-75)$ & $50(25-75)$ & $50(25-75)$ & $0.54^{*}$ \\
\hline Dolor corporal & $55.5(41-72)$ & $74(50.25-84)$ & $61(50-76)$ & $0.04^{*}$ \\
\hline Salud general & $61(45-80.25)$ & $65(47-79.5)$ & $60(50-72.5)$ & $0.64^{*}$ \\
\hline Vitalidad & $75(70-88.75)$ & $80(70-90)$ & $75(61.5-85)$ & $0.03^{*}$ \\
\hline Función social & $68.5(50-100)$ & $92(62-100)$ & $81(62-100)$ & $0.32^{*}$ \\
\hline Rol emocional & $100(69-100)$ & $100(66-100)$ & $100(76-100)$ & $0.72^{*}$ \\
\hline Salud mental & $84(75-95)$ & $88(76-96)$ & $88(68-92)$ & $0.29^{*}$ \\
\hline
\end{tabular}

Todos los valores están expresados en mediana y amplitud intercuartil.

EVA: escala visual analógica.

*Kruskal-Wallis.

Tabla 4. Análisis de resultados mediante un modelo de regresión logística binaria

\begin{tabular}{lccccccc}
\hline Paso 4 & B & ES & Wald & gl & p & RM & IC 95\% \\
\hline Ansiedad & 2.062 & 0.545 & 14.301 & 1 & $<0.001$ & 7.9 & $2.7-23$ \\
$\begin{array}{l}\text { Función } \\
\text { social }\end{array}$ & 0.024 & 0.011 & 4.96 & 1 & 0.026 & 1.024 & $1.003-1.046$ \\
Vitalidad & 0.057 & 0.017 & 11.882 & 1 & $<0.001$ & 1.059 & $1.025-1.094$ \\
Salud & 0.074 & 0.016 & 22.153 & 1 & $<0.001$ & 1.077 & $1.044-1.111$ \\
mental & & & & & & & \\
Constante & -11.951 & 1.679 & 50.635 & 1 & $<0.001$ & & \\
\hline
\end{tabular}

Se tomó como referencia para el análisis la presencia o ausencia de depresión. RM: razón de momios; IC 95\%: intervalo de confianza del 95\%.

Igualmente, Duivenvoorden, et al..$^{15}$ observaron que la depresión y la ansiedad podrían disminuir a 1 año de haberse realizado la ATR, pero que en sujetos con ansiedad y depresión preoperatoria se tienen peores resultados funcionales y mayor insatisfacción.

En este estudio, como pudo observarse, los pacientes que se clasificaron con depresión tuvieron puntuaciones bajas en todos los dominios del CSF-36, en oposición con Pérez-Prieto, et al., ${ }^{25}$ quienes reportan que los sujetos con depresión y ATR no tienen afección de la puntuación de los componentes del CSF-36 tras 5 años de una ATR, y que por ello la depresión no debe contraindicar la ATR. Esta diferencia puede explicarse porque en ese estudio no se tomó en cuenta la ansiedad, que en nuestro reporte fue un factor predictor independiente asociado a depresión en ancianos tras 5 años de ser operados de ATR. Por lo tanto, detectar los pacientes con depresión y ansiedad, para ser tratados de ellas antes de operarse, debe ser parte integral de los protocolos de atención primaria en pacientes con gonartrosis para potenciar los beneficios de la ATR.

Otro factor que nos parece relevante mencionar es el estado comórbido. En un estudio ${ }^{26}$ se refiere que las alteraciones psiquiátricas, la hipertensión arterial sistémica y la diabetes son factores que se asocian a cirugía de revisión de la ATR en los primeros 5 años después de la operación primaria. No obstante, en este reporte, los sujetos diabéticos e hipertensos mostraron poca afección en la CV. Resultados parecidos se obtuvieron en otro estudio ${ }^{27}$, en el que los sujetos diabéticos no mostraron disminución en las puntuaciones de los dominios del CSF-36, lo que muestra que la diabetes no debe contraindicar una ATR. Sin embargo, es evidente que los estados comórbidos pueden asociarse con afecciones del estado mental, y la presencia de comorbilidad en sujetos candidatos a ATR podría ser un factor asociados a depresión; por esta razón, los sujetos con comorbilidad deben ser evaluados en profundidad, porque podrían continuar con dolor y tener malos resultados funcionales después de la ATR $^{28}$.

Por otro lado, en este reporte se observó que las mujeres se sometieron en mayor proporción a ATR y presentaron con más frecuencia depresión y ansiedad. Buller, et al. ${ }^{29}$ refieren resultados similares a los nuestros, con un $78 \%$ y un $76 \%$ de mujeres con depresión y ansiedad, respectivamente. Esto puede deberse a la mayor esperanza de vida de las mujeres y a que en ellas son más prevalentes los trastornos mentales.

En cuanto al análisis de la CV en los sujetos con obesidad y ATR, en el estudio de Xu, et al. ${ }^{13}$ se refiere que los sujetos con obesidad mejoran su CV en el 
posoperatorio, pero al ser evaluados luego de 2 y 10 años muestran puntuaciones decrecientes en todos los dominios del CSF-36. Sin embargo, en este reporte se observó que los sujetos con un IMC normal presentaron puntuaciones bajas en más componentes del CSF-36 en comparación con los obesos, lo que concuerda con un reporte que sustenta que la CV y la función en los obesos, luego de 5 años de una ATR, no son diferentes de las de los sujetos de peso normal ${ }^{30}$, pero que los sujetos obesos presentan puntuaciones más altas en la intensidad del dolor. De manera similar, nosotros observamos en la EVA puntuaciones más altas en la percepción de dolor en los obesos, pero con escasa afección de los componentes de CV. En contraste, un estudio ${ }^{31}$ refiere que los sujetos obesos, a 6 meses del posoperatorio de ATR, tienen una intensidad del dolor similar a la de los sujetos sin obesidad. De igual manera, Jones, et al. ${ }^{32}$ señalan que, después de 3 años de una ATR, no existen diferencias en cuanto al dolor en obesos y en los pacientes con menor IMC, y que no debe contraindicarse la ATR en individuos obesos. Por lo anterior, persiste la interrogante de si debe negarse o no la ATR a los sujetos obesos. Es de importancia señalar que nosotros estudiamos pacientes con más de 5 años de operados y que la edad mediana fue de 71 años, lo que refleja que la edad afecta la CV y podría aumentar los niveles de dolor en los ancianos obesos con ATR.

Como limitaciones del estudio cabe mencionar que no se documentaron el IMC, la depresión, la ansiedad ni la CV antes de la ATR, lo que impide evaluar posibles cambios. No se aplicó una escala de evaluación funcional para comparar los resultados en los sujetos obesos, así como en los sujetos con y sin depresión. Tampoco se documentó el riesgo anestésico para evaluar la afección orgánica en los pacientes con comorbilidad asociada a la gonartrosis. El estudio se circunscribió a pacientes de una sola región geográfica, lo que puede dificultar la generalización de los resultados. No se confirmó el diagnóstico de depresión por un psiquiatra, lo que pudo causar un sesgo de clasificación.

Entre las fortalezas del estudio destaca el uso de la escala CENASEM, creada y validada para la población anciana mexicana. El muestreo fue probabilístico para disminuir posibles sesgos de selección. Las entrevistas fueron ciegas e independientes, y los encuestadores fueron personal ajeno a la investigación para evitar sesgos de cortesía y del entrevistador.

\section{Conclusión}

Los sujetos con depresión presentan puntuaciones bajas en los componentes del CSF-36 y reportan puntuaciones altas en la percepción del dolor en la EVA. La ansiedad fue el factor predictor más importante, asociado a depresión en sujetos ancianos con ATR. Del mismo modo, los componentes del CSF-36 que son posibles factores asociados a depresión en sujetos con más de 5 años de haber sido operados de ATR son la función social, la vitalidad y la salud mental. La obesidad en los sujetos con ATR aumenta la percepción del dolor, con escasa afección en la CV. Nosotros recomendamos hacer una evaluación sistemática con cuestionarios como el CENASEM y el CSF-36 en ancianos que se someterán a ATR, dado que detectar, tratar y dar seguimiento a la depresión podría aumentar la CV y mejorar los resultados funcionales de la ATR. Finalmente, en los ancianos con obesidad y ATR recomendamos hacer más trabajos de investigación, ya que la evidencia aún es discordante.

\section{Conflicto de intereses}

Los autores declaran no tener ningún conflicto de intereses.

\section{Responsabilidades éticas}

Protección de personas y animales. Los autores declaran que para esta investigación no se han realizado experimentos en seres humanos ni en animales.

Confidencialidad de los datos. Los autores declaran que han seguido los protocolos de su centro de trabajo sobre la publicación de datos de pacientes.

Derecho a la privacidad y consentimiento informado. Los autores han obtenido el consentimiento informado de los pacientes y/o sujetos referidos en el artículo. Este documento obra en poder del autor de correspondencia.

\section{Bibliografía}

1. Yang Z, Liu H, Xie X, Tan Z, Qin T, Kang P. The influence of diabetes mellitus on the post-operative outcome of elective primary total knee replacement: a systematic review and meta-analysis. Bone Joint J. 2014;96-B:1637-43.

2. Dusad A, Pedro S, Mikuls TR, Hartman CW, Garvin KL, O'Dell JR, et al. Impact of total knee arthroplasty as assessed using patient-reported pain and health-related quality of life indices: rheumatoid arthritis versus osteoarthritis. Arthritis Rheumatol. 2015;67:2503-11.

3. Weinstein AM, Rome BN, Reichmann WM, Collins JE, Burbine SA, Thornhill TS, et al. Estimating the burden of total knee replacement in the United States. J Bone Joint Surg Am. 2013;95:385-92.

4. Maradit Kremers $H$, Larson DR, Crowson CS, Kremers WK, Washington RE, Steiner CA, et al. Prevalence of total hip and knee replacement in the United States. J Bone Joint Surg Am. 2015;97:1386-97. 


\section{S. Charles-Lozoya, et al.: Calidad de vida en prótesis de rodilla}

5. Peter WF, Dekker J, Tilbury C, Tordoir RL, Verdegaal SH, Onstenk R, et al. The association between comorbidities and pain, physical function and quality of life following hip and knee arthroplasty. Rheumatol Int. 2015;35:1233-41.

6. Smith TO, Penny F, Fleetcroft R. Medical morbidities in people following hip and knee arthroplasty: data from the Osteoarthritis Initiative. Eur J Orthop Surg Traumatol. 2016;26:99-106.

7. Hossain FS, Konan S, Patel S, Rodríguez-Merchán EC, Haddad FS. The assessment of outcome after total knee arthroplasty: are we there yet? Bone Joint J. 2015;97-B:3-9

8. Bierke S, Häner M, Petersen W. Influence of somatization and depressive symptoms on the course of pain within the first year after uncomplicated total knee replacement: a prospective study. Int Orthop. 2016;40:1353-60.

9. Browne JA, Sandberg BF, D'Apuzzo MR, Novicoff WM. Depression is associated with early postoperative outcomes following total joint arthroplasty: a nationwide database study. J Arthroplasty. 2014;29:481-3.

10. Riddle DL, Wade JB, Jiranek WA. Major depression, generalized anxiety disorder, and panic disorder in patients scheduled for knee arthroplasty. J Arthroplasty. 2010;25:581-8.

11. Qi A, Lin C, Zhou A, Du J, Jia X, Sun L, et al. Negative emotions affect postoperative scores for evaluating functional knee recovery and quality of life after total knee replacement. Braz J Med Biol Res. 2016;49:e4616.

12. Chen JY, Lo NN, Chong HC, Bin Abd Razak HR, Pang HN, Tay DK et al. The influence of body mass index on functional outcome and quality of life after total knee arthroplasty. Bone Joint J. 2016;98-B:780-5.

13. Xu S, Chen JY, Lo NN, Chia SL, Tay DKJ, Pang HN, et al. The influence of obesity on functional outcome and quality of life after total knee arthroplasty: a ten-year follow-up study. Bone Joint J. 2018;100-B:579-83.

14. Kandil A, Novicoff WM, Browne JA. Obesity and total joint arthroplasty: do patients lose weight following surgery? Phys Sportsmed. 2013;41:34-7.

15. Duivenvoorden T, Vissers MM, Verhaar JA, Busschbach JJ, Gosens T, Bloem RM, et al. Anxiety and depressive symptoms before and after total hip and knee arthroplasty: a prospective multicentre study. Osteoarthritis Cartilage. 2013;21:1834-40.

16. Aguilar-Navarro SG, Fuentes-Cantú A, Ávila-Funes JA, García-Mayo EJ Validity and reliability of the screening questionnaire for geriatric depression used in the Mexican Health and Age Study. Salud Publica Mex. 2007;49:256-62.

17. Zúniga MA, Carrillo-Jiménez GT, Fos PJ, Gandek B, Medina-Moreno MR Evaluation of health status using Survey SF-36: preliminary results in Mexico. Salud Publica Mex. 1999;41:110-8.

18. Hawker GA, Mian S, Kendzerska T, French M. Measures of adult pain Visual Analog Scale for Pain (VAS Pain), Numeric Rating Scale for Pain (NRS Pain), McGill Pain Questionnaire (MPQ), Short-Form McGill Pain Questionnaire (SF-MPQ), Chronic Pain Grade Scale (CPGS), Short Form-36 Bodily Pain Scale (SF-36 BPS), and Measure of Intermittent and Constant Osteoarthritis Pain (ICOAP). Arthritis Care Res (Hoboken). 2011;63(Suppl 11):S240-52.
19. Goldberg D, Bridges K, Duncan-Jones P, Grayson D. Detecting anxiety and depression in general medical settings. BMJ. 1988;297:897-9.

20. Stundner O, Kirksey M, Chiu YL, Mazumdar M, Poultsides L, Gerner P, et al. Demographics and perioperative outcome in patients with depression and anxiety undergoing total joint arthroplasty: a population-based study. Psychosomatics. 2013;54:149-57.

21. Singh JA, Lewallen DG. Time trends in the characteristics of patients undergoing primary total knee arthroplasty. Arthritis Care Res (Hoboken). 2014;66:897-906.

22. Dhurve K, Scholes C, El-Tawil S, Shaikh A, Weng LK, Levin K, et al. Multifactorial analysis of dissatisfaction after primary total knee replacement. Knee. 2017;24:856-62.

23. Bistolfi A, Bettoni E, Aprato A, Milani P, Berchialla P, Graziano E, et al. The presence and influence of mild depressive symptoms on post-operative pain perception following primary total knee arthroplasty. Knee Surg Sports Traumatol Arthrosc. 2017;25:2792-800.

24. Khatib $Y$, Jenkin D, Naylor JM, Harris IA. Psychological traits in patients waiting for total knee arthroplasty. A cross-sectional study. J Arthroplasty. 2016;31:1661-6.

25. Pérez-Prieto D, Gil-González S, Pelfort X, Leal-Blanquet J, Puig-Verdié L, Hinarejos $P$. Influence of depression on total knee arthroplasty outcomes. J Arthroplasty. 2014;29:44-7.

26. Jämsen E, Peltola M, Eskelinen A, Lehto MU. Comorbid diseases as predictors of survival of primary total hip and knee replacements: a nationwide register-based study of 96754 operations on patients with primary osteoarthritis. Ann Rheum Dis. 2013;72:1975-82.

27. Singh JA. Effect of comorbidity on quality of life of male veterans with prevalent primary total knee arthroplasty. Clin Rheumatol. 2009;28: 1083-9.

28. Utrillas-Compaired A, De la Torre-Escuredo BJ, Tebar-Martínez AJ, Asúnsolo-Del Barco A. Does preoperative psychologic distress influence pain, function, and quality of life after TKA? Clin Orthop Relat Res. 2014:472:2457-65.

29. Buller LT, Best MJ, Klika AK, Barsoum WK. The influence of psychiatric comorbidity on perioperative outcomes following primary total hip and knee arthroplasty; a 17-year analysis of the National Hospital Discharge Survey database. J Arthroplasty. 2015;30:165-70.

30. Torres-Claramunt R, Hinarejos P, Leal-Blanquet J, Sánchez-Soler JF, Marí-Molina R, Puig-Verdié L, et al. Does obesity influence on the functional outcomes of a total knee arthroplasty? Obes Surg. 2016;26: 2989-94.

31. Li W, Ayers DC, Lewis CG, Bowen TR, Allison JJ, Franklin PD. Functional gain and pain relief after total joint replacement according to obesity status. J Bone Joint Surg Am. 2017;99:1183-9.

32. Jones CA, Cox V, Jhangri GS, Suárez-Almazor ME. Delineating the impact of obesity and its relationship on recovery after total joint arthroplasties. Osteoarthritis Cartilage. 2012;20:511-8. 